\title{
A low complexity uplink multiuser MIMO detecting algorithm based on BQRD
}

\author{
MA Jun, WANG Da-ming \\ Institute of information and System Engineering \\ PLA Information Engineering University \\ Zhengzhou, China \\ e-mail: majun0316@126.com
}

\begin{abstract}
In multiuser MIMO uplink communications, it is necessary to design linear schemes that are able to suppress cochannel interferences(CCIs). Block diagonalization(BD), which can achieve good CCIs suppression, has been extensively studied to solve the issue. However, with the scheme we cannot obtain extra diversity gain due to the null steering at the base station. In this paper, we propose a linear block QR decomposition(BQRD) which transforms the multiuser channel to a block upper triangular structure based on GramSchmidt orthonormalization and can obtain more diversity gain than BD. Its error rate performance is evaluated using computer simulations. The result shows that, the proposed scheme provides excellent performance with low complexity. A optimal ordering criterion is proposed at last to do full study of BQRD scheme. Simulation results show that the BQRD with the optimal ordering proposed achieves much better performance than the non-ordering one.
\end{abstract}

Keywords- uplink multiuser MIMO system; Co-Channel Interferences; multiuser channel matrix decomposition

\section{INTRODUCTION}

Multiple-input Multiple-output (MIMO) system has been extensively studied 错误! 未找到引用源。[1] as a core technology to achieve high data-rate wireless communications over a limited frequency resource. Recently, Multiuser MIMO(MU-MIMO) system based on the concept of space division multiple access has also attracted much attention ${ }^{[2]}$. MU-MIMO systems are impaired with Co-Channel Interferences(CCIs) due to the frequency reuse, multiple access schemes and multiuser communications. Some nonlinear algorithms have been proposed to solve the problem 错误! 未找到引用源。[3][4][5], for example Dirty paper coding(DPC) 错误!未找到引用源。 [5]. But in the future wireless communi-cation system, the combination of MIMO and OFDM will bring huge Computation, which makes nonlinear algorithms with high complexity hardly to be used in practice. As a result, the study on low complexity linear detection algorithms has great value.

One of the linear solutions for the CCIs suppression is block diagonalization(BD) 错误!未找到引用源。 [6][7]. In this scheme, the multiuser MIMO channel can be decomposed to singleuser MIMO channels without CCIs. To achieve such decomposition, the number of receive antennas of base station must not be less than the sum of the transmit antennas for all users. Thus, in the BD system, most of the spatial resources (in other words, the number of receive antennas) are consumed to direct nulls to all antenna elements of undesired users even if full spatial multiplexing in each single-user-MIMO(SU-MIMO) channels is never used. In such a case, it can be said that the BD system loses the potential for transmit-diversity gain. On the other hand, BD decomposes the MU-MIMO system into parallel SUMIMO systems, so that SU-MIMO based algorithms must be used in detection, which make it hardly to move forward to reduce the complexity.

One brightly method named block QR decomposition (BQRD) 错误!未找到引用源。 ${ }^{88]}$ is proposed to suppress CCIs. But to our knowledge, there are at least two issues have not been answered. One is how to apply this algorithm to the uplink multiuser MIMO System, which is without precoding ${ }^{[2]}$. The other is BQRD in Ref.[8] should combine with DPC, which make it hardly to be used in practice. These will be the main topics in the following sections.

The rest of this paper is organized as follows. Section II describes the uplink multiuser MIMO system handled in our study. Section III explains the proposed linear algorithm based on BQRD and analyzes its complexity. Section IV explains the optimal ordering scheme of the BQRD. Section $\mathrm{V}$ analyzes the performance of these schemes by computer simulations, and finally conclusions are drawn in Section VI.

\section{SYSTEM MODEL}

Let us consider the uplink multiuser MIMO system where the base station has $M$ antennas, each mobile station (user) has $N_{k}$ antennas 错误!未找到引用源。[9]. The number of existing mobile stations is $K$. Note that we assume $M \geq K \times N_{k}$. It is also assumed that the transmission frequency bandwidth is so narrow that the fading is frequency-flat. A $M \times N_{k}$ channel matrix of the $k$ th user is defined as $\mathbf{H}_{k}$, which is the Rayleigh block fading channel. Each entry in the channel matrix is modeled as a statistically independent and identically distributed zero-mean complex Gaussian variable with unit varian-ce. The signals sent over $N_{k}$ transmit antennas by the $k$ th user is represented as

$$
\mathbf{x}_{k}=\left[\begin{array}{llll}
x_{k 1} & x_{k 2} & \cdots & x_{k N_{k}}
\end{array}\right]^{T}
$$

where $x_{k 1}, \cdots, x_{k N_{k}}$ have unit energy. signal samples on those $M$ receive antennas are expressed as 


$$
\mathbf{Y}=\sum_{k=1}^{K} \mathbf{H}_{k} \mathbf{x}_{k}+\mathbf{Z}
$$

furthermore, the additive noise $\mathbf{Z}$ vector is assumed to have independent complex Gaussian elements with variance $\sigma^{2}$ and is spatially white, i.e.,

$$
\mathrm{E}\left[\mathbf{Z Z}^{*}\right]=\sigma^{2} \mathbf{I}_{M}
$$

where $\mathbf{I}_{M}$ is the $M \times M$ identity matrix. Since the random quantities $\mathbf{H}_{k}, \mathbf{x}_{k}, \mathbf{Z}$ are assumed independent, we shall plot all the BER curves in this paper versus $1 / \sigma^{2}$. The quantity $1 / \sigma^{2}$ represents the SNR per receive antenna for uplink multiuser MIMO systems without precoding.

\section{BLOCK QR DECOMPOSITION}

In this section, we generalise the Gram - Schmidt method to implement Block QR Decomposition in uplink MUMIMO. The channel matrix of a single user is projection to the orthogonal space of processed channel matrices through the Gram - Schmidt method. Then uplink multiuser channel matrix is decomposed into an orthogonal matrix and a block upper triangular matrix. And multiuser CCIs is migrated trough cancellation. The linear BQRD algorithm is implemented using the following steps.

Firstly, we introduce the way to generalise the Gram Schmidt method. The multiuser channel matrix is given as $\mathbf{H}=\left[\begin{array}{llll}\mathbf{H}_{1} & \mathbf{H}_{2} & \cdots & \mathbf{H}_{K}\end{array}\right]$. Then, to $k$ th user, we use $\mathbf{H}_{k}$, for $k=1, \cdots, K$, to compute $\overline{\mathbf{H}}_{k}$ as

$$
\overline{\mathbf{H}}_{k}=\left(\mathbf{I}-\sum_{i=1}^{k-1} \mathbf{U}_{i} \mathbf{U}_{i}^{H}\right) \mathbf{H}_{k}
$$

where $\mathbf{U}_{i}$ is obtained from the SVD of $\overline{\mathbf{H}}_{i}$, that is,

$$
\overline{\mathbf{H}}_{i}=\left(\begin{array}{ll}
\mathbf{U}_{i} & \mathbf{W}_{i}
\end{array}\right)\left(\begin{array}{c}
\mathbf{D}_{i} \\
0
\end{array}\right) \mathbf{V}_{i}^{H}=\mathbf{U}_{i} \mathbf{D}_{i} \mathbf{V}_{i}^{H}
$$

the columns of $\mathbf{U}_{i}$ are left singular vectors corresponding to the non-zero singular values of $\overline{\mathbf{H}}_{i}$. And $\mathbf{D}_{i}$ only contains the non-zero singular values of $\overline{\mathbf{H}}_{i}$ as diagonal elements.

Using the SVD of $\overline{\mathbf{H}}_{k}$, we can obtain

$$
\begin{aligned}
\mathbf{H}_{k} & =\overline{\mathbf{H}}_{k}+\sum_{i=1}^{k-1} \mathbf{U}_{i} \mathbf{U}_{i}^{H} \mathbf{H}_{k} \\
& =\left(\begin{array}{llllllll}
\mathbf{U}_{1} & \cdots & \mathbf{U}_{k-1} & \mathbf{U}_{k} & \mathbf{U}_{k+1} & \cdots & \mathbf{U}_{K}
\end{array}\right) \\
& \bullet\left(\begin{array}{llllllll}
\mathbf{U}_{1}^{H} \mathbf{H}_{k} & \cdots & \mathbf{U}_{k-1}^{H} \mathbf{H}_{k} & \mathbf{D}_{k} \mathbf{V}_{k}^{H} & 0 & \cdots & 0
\end{array}\right)^{T}
\end{aligned}
$$

As a result, we obtain the BQRD structure as

$$
\mathbf{H}=\left[\begin{array}{llll}
\mathbf{U}_{1} & \mathbf{U}_{2} & \cdots & \mathbf{U}_{K}
\end{array}\right]\left[\begin{array}{cccc}
\mathbf{D}_{1} \mathbf{V}_{1}^{H} & \mathbf{U}_{1}^{H} \mathbf{H}_{2} & \cdots & \mathbf{U}_{1}^{H} \mathbf{H}_{K} \\
& \mathbf{D}_{2} \mathbf{V}_{2}^{H} & & \mathbf{U}_{2}^{H} \mathbf{H}_{K} \\
& & \ddots & \vdots \\
& & & \mathbf{D}_{K} \mathbf{V}_{K}^{H}
\end{array}\right]
$$

(7)

the left singular matrix $\mathbf{U}_{k}$ is used to construct the unitary matrix $\mathbf{Q}$ as $\mathbf{Q}=\left[\begin{array}{llll}\mathbf{U}_{1} & \mathbf{U}_{2} & \cdots & \mathbf{U}_{K}\end{array}\right]$.

Then, we will discuss how to migrate multiuser CCIs through cancellation with BQRD results. From (2), the signals received in the base station can be translated into

$$
\mathbf{Y}=\mathbf{H X}+\mathbf{Z}
$$

where $\mathbf{X}=\left[\begin{array}{llll}\mathbf{x}_{1} & \mathbf{x}_{2} & \cdots & \mathbf{x}_{K}\end{array}\right]^{T}, \mathbf{x}_{k}$ represent the signals sent over $N_{k}$ transmit antennas by the $k$ th user.

So, we deal with received signals $\mathbf{Y}$, using the result of BQRD as follows,

$$
\begin{aligned}
\overline{\mathbf{Y}} & =\mathbf{Q}^{H} \mathbf{Y}=\mathbf{Q}^{H} \mathbf{H} \mathbf{X}+\mathbf{Q}^{H} \mathbf{Z} \\
& =\left[\begin{array}{cccc}
\mathbf{D}_{1} \mathbf{V}_{1}^{H} & \mathbf{U}_{1}^{H} \mathbf{H}_{2} & \cdots & \mathbf{U}_{1}^{H} \mathbf{H}_{K} \\
& \mathbf{D}_{2} \mathbf{V}_{2}^{H} & & \mathbf{U}_{2}^{H} \mathbf{H}_{K} \\
& & \ddots & \vdots \\
& & & \mathbf{D}_{K} \mathbf{V}_{K}^{H}
\end{array}\right]\left[\begin{array}{c}
\mathbf{x}_{1} \\
\mathbf{x}_{2} \\
\vdots \\
\mathbf{x}_{K}
\end{array}\right]+\overline{\mathbf{Z}}
\end{aligned}
$$

where $\overline{\mathbf{Y}}=\left[\begin{array}{llll}\overline{\mathbf{y}}_{1} & \overline{\mathbf{y}}_{2} & \cdots & \overline{\mathbf{y}}_{K}\end{array}\right]^{T}, \overline{\mathbf{y}}_{k}$ correspond to $\mathbf{x}_{k}$.

Thus, the following process is described as follows:

First for the $K$ th user

$\overline{\mathbf{x}}_{K}=\mathbf{G}_{K} \overline{\mathbf{y}}_{K}$

where $\mathbf{G}_{K}=\mathbf{V}_{K} \mathbf{D}_{K}^{-1}$

for $\mathrm{i}=K-1$ :descend: 1

$\hat{\mathbf{y}}_{i}=\overline{\mathbf{y}}_{i}$

for $\mathrm{j}=\mathrm{i}+1$ : $K$

$\hat{\mathbf{y}}_{i}=\hat{\mathbf{y}}_{i}-\mathbf{U}_{i}^{H} \mathbf{H}_{j} \overline{\mathbf{x}}_{j}$

end

$\overline{\mathbf{x}}_{i}=\mathbf{G}_{i} \hat{\mathbf{y}}_{i}$

where $\mathbf{G}_{i}=\mathbf{V}_{i} \mathbf{D}_{i}^{-1}$

end

output $\overline{\mathbf{x}}_{1}, \quad \overline{\mathbf{x}}_{2}, \cdots, \overline{\mathbf{x}}_{K}$

From discussions above, we can see the Equivalent channel matrix $\overline{\mathbf{H}}_{k}$ is consumed to direct nulls to Equivalent channel matrices $\overline{\mathbf{H}}_{i}, i=1, \cdots, k-1$ of the users, which have already been processed. Thus, the signals of $k$ th user loses more transmit-diversity gain than the users processed in front.

The Complexity of linear BQRD will be discussed in compared with BD. The complexity of computing the inverse or the eigenvalues of an $q \times q$ Hermitian matrix is

$O\left(q^{w}\right), 2<w<3$. The complexity of computing SVD decomposition of a $q \times p$ matrix is $O\left(\max \left(p q^{2}, p^{2} q, q^{3}\right)\right)$. So, the main complexity of linear BQRD is about $K O\left(M^{3}\right)$. However, the main complexity of BD combined with simplest SU-MIMO detection algorithm Zero Forcing(ZF) is about $K O\left(M^{3}\right)+K O\left(N^{w}\right) 2<w<3$. So we can say linear $\mathrm{BQRD}$ has an advantage over BD in complexity. 


\section{ORDERING SCHEME WITH WATER FILLING}

For linear BQRD algorithm, the processing sequence of user bring differences in performance. Therefore, to find the optimal sequence of processing is a problem worthy of study.To our knowledge, $K$ will not be a large number in the future LTE-Advanced wireless communication system. So we just consider the exhaustive method with best performance regardless of fast methods.

From the discussion in section III, the last user processed in BQRD suffer the maximum loss of transmit-diversity gain, which is decided by its nature. The user of ill-condition cannot be put to the last position of BQRD, otherwise it will not achieve acceptable results of detection. With the Waterfilling method and sigular value decomposition of channel matrices, we can achieve maximum data rate of each user. Then, the linear BQRD will be done according to increasing sequence of maximum data rate of each user.

\section{SIMULATIONS}

Computer simulations are conducted to verify the proposed criterion and algorithm. System performance is measured in terms of Bit Error Rate(BER)with a flame of 128 QPSK symbols and averaged over 5000 MIMO channel realizations. The channel is assumed to be Rayleigh block fading which is independent from one frame to the other and unchanged during one frame. we set the number of transmit antennas of each user to $N_{k}=2$ and the number of users to $K=3$. The number of receive antennas in base station is $M=6$.

Figure 1 presents the performance comparisons between BD algorithm and BQRD algorithm in the same fading environment. Comparing with the $\mathrm{BD}+\mathrm{ZF}$ scheme, the BQRD obtains about $3 \mathrm{~dB}$ gains. This is because that in the $\mathrm{BD}$ system, most of the spatial resources are consumed to direct nulls to all antenna elements of undesired users. The figure also shows that the BQRD-based scheme has the similar performance with BD +MMSE scheme, which needs to know the variance of the noise in base station.

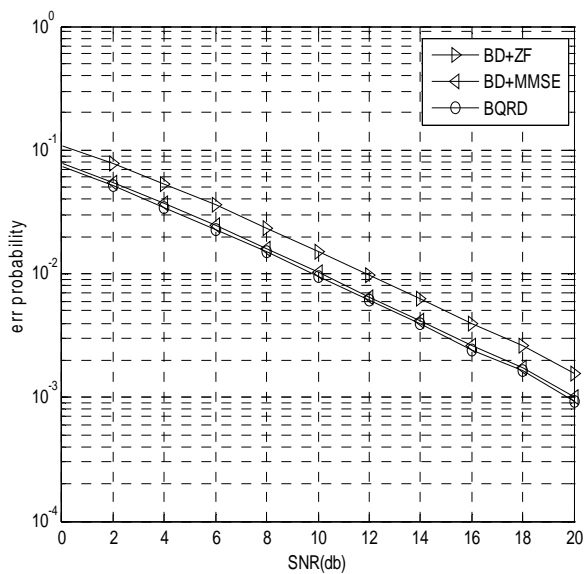

Figure 1. BER performance comparisons between BD and BQRD
Figure 2 shows the performance of different users using BQRD algorithm. From the discussion in section III, we know Equivalent channel matrix of the user is consumed to direct nulls to Equivalent channel matrices of the users, which have already been processed. Thus, the signals of the 3 rd user loses more transmit-diversity gain than the users processed in front. And the 2 nd user loses more transmit-diversity gain than the 1 st one. The simulating result is consistent well with the theoretical estimate.

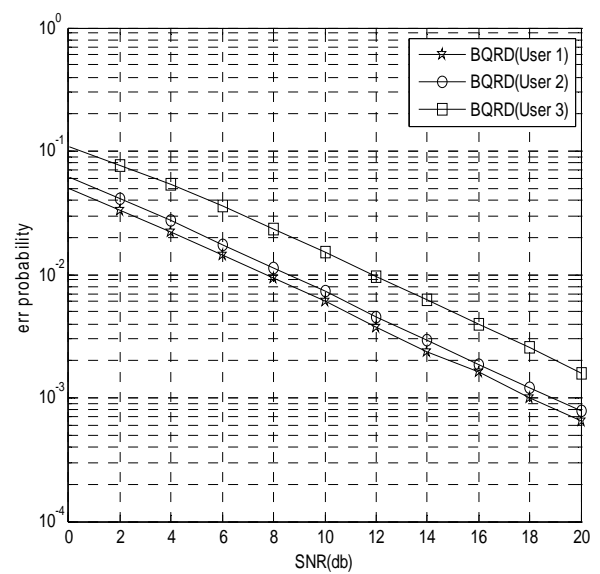

Figure 2. BER performance of different users in BQRD scheme

The comparison of time consumption for detection between BQRD and BD scheme is shown in figure 3, for which a computer with an Intel(R) Core(TM) i5-3210M CPU and the Matlab7.10.0 software were used in our simulations. The simulation results show that BQRD save much time as times of simulation increases. In the future wireless communication system, the OFDM tecnology will bring huge Computation burden. As running time of system increases, it will save much computing resources with BQRD scheme.

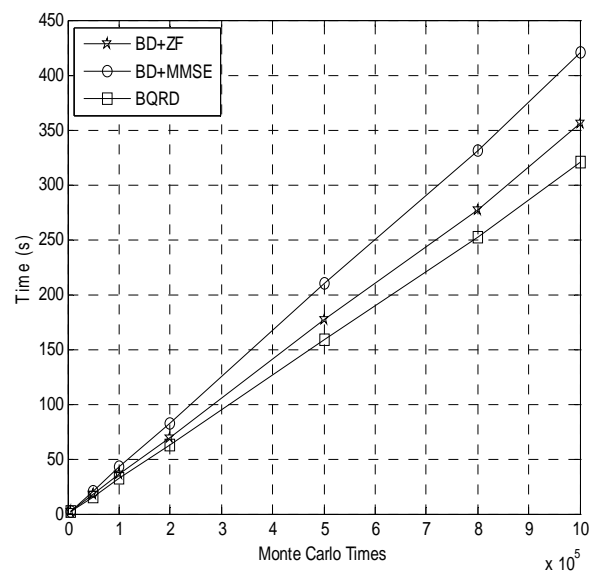

Figure 3. Monte Carlo Times for the three schemes

Figure 4 presents the performance comparisons between 
the exhaustive ordering and the non-ordering one. From the figure, we can observe that the system with user selection outperforms the system without ordering obviously. The simulating result is consistent well with the theoretical estimate in section IV.

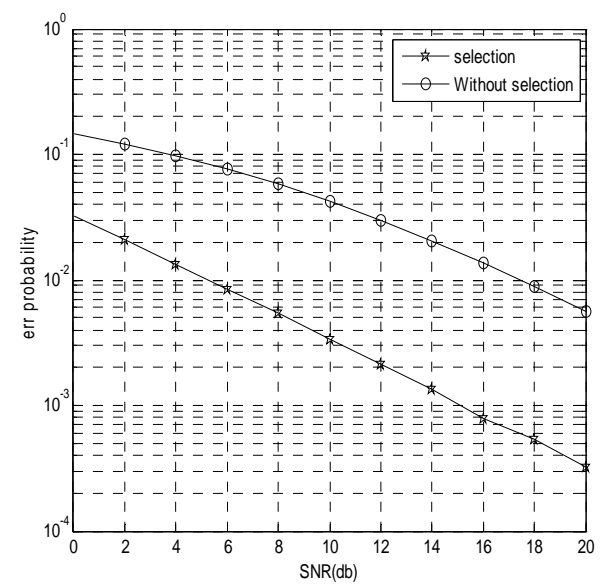

Figure 4. BER performance comparisons between exhaustive ordering and non-ordering

\section{CONCLUSIONS}

In this paper, we have proposed linear block QR decomposition for multiuser MIMO uplink system and evaluated its performance by computer simulations. The results showed that the proposed scheme outperforms the conventional block diagonalization especially in its low computational complexity. We have also take the ordering of BQRD into consideration, which achieves much better performance than the non-ordering one. Our future work involves studies on multiuser system combined with the Space-Time Coding, performance evaluation using system capacity and theoretical analysis of the proposed BQRD system.

\section{ACKNOWLEDGMENT}

Work for this article was supported by the National High-Tech Research and Development Plan of China (2012AA01A502, 2012AA01A505)

\section{REFERENCES}

[1] E. Telatar, “Capacity of multi-antenna Gaussian channels,” European Transactions on Telecommunications, vol. 10, no.6,1999,pp.585-595.

[2] Quentin H.Spencer, Christian B.Peel, A.Lee Swindlehurst, Martin Haardt, “An Introduction to the Multi-User MIMO Downlink,” IEEE Communications Magazine, vol.42, no.10, 2004, pp.60-67.

[3] M.Costa, “Writing on Dirty Paper," IEEE Trans. Info. Theory, vol. 29,no. 3, May, 1983, pp. 439-41.

[4] CHEN S, HANZO L. "Minimum bit-error rate design for space-time equalization-based multiuser detection," IEEE Trans on Communication, vol.54, no.5, 2006, pp.824-831.

[5] QIN Z L, CAI K, ZOU X X, “Turbo multiuser detection based on local search algorithms," IEEE Proceedings in the Internet Conf on Commun, Singapore, 2007, pp.5987-5992.

[6] M. Rim, "Multi-user downlink beamforming with multiple transmit and receive antennas,” Electron. Lett., vol. 38, no. 25, Dec. 2002, pp. 1725-1726.

[7] L.U. Choi and R.D. Murch, "A transmit preprocessing technique for multiuser MIMO systems using a decomposition approach,” IEEE Trans.Wireless Commun., vol. 3, no. 1, Jan. 2004, pp. 20-24.

[8] A.K. Yousafzai and M.R. Nakhai, "Block QR decomposition and near-optimal ordering in intercell cooperative multiple-input multipleoutput-orthogonal frequency division multiplexing," IET Commun., Vol. 4, Iss. 12, 2010, pp. 1452-1462

[9] M. Rim, "Multi-user downlink beamforming with multiple transmit and receive antennas,” Electron. Lett., vol. 38, no. 25, Dec. 2002, pp. 1725-1726, 